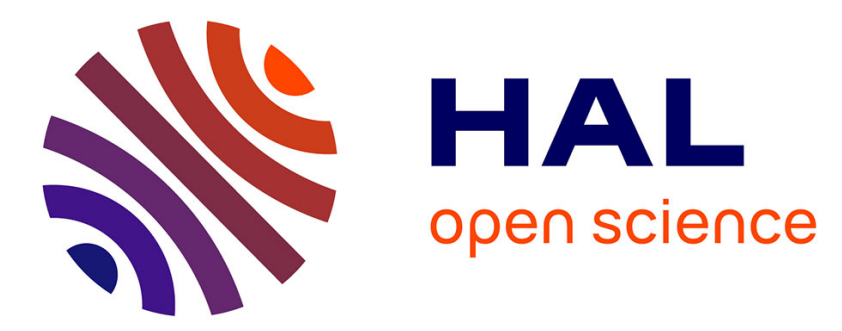

\title{
Event-based control strategy for consensus of a group of VTOL-UAVs
}

\author{
A. Vega-Alonzo, Jose Fermi Guerrero Castellanos, Sylvain Durand, Nicolas \\ Marchand, S. E. Maya-Rueda, Gerardo Mino-Aguilar
}

\section{To cite this version:}

A. Vega-Alonzo, Jose Fermi Guerrero Castellanos, Sylvain Durand, Nicolas Marchand, S. E. MayaRueda, et al.. Event-based control strategy for consensus of a group of VTOL-UAVs. EBCCSP 2016 - 2nd IEEE Conference on Event-Based Control Communication and Signal Processing, Jun 2016, Cracovie, Poland. hal-01330792

\section{HAL Id: hal-01330792 https://hal.science/hal-01330792}

Submitted on 13 Jun 2016

HAL is a multi-disciplinary open access archive for the deposit and dissemination of scientific research documents, whether they are published or not. The documents may come from teaching and research institutions in France or abroad, or from public or private research centers.
L'archive ouverte pluridisciplinaire HAL, est destinée au dépôt et à la diffusion de documents scientifiques de niveau recherche, publiés ou non, émanant des établissements d'enseignement et de recherche français ou étrangers, des laboratoires publics ou privés. 


\title{
Event-based control strategy for consensus of a group of VTOL-UAVs
}

\author{
A. Vega-Alonzo*, J.F. Guerrero-Castellanos*, S. Durand ${ }^{\ddagger}$, N. Marchand ${ }^{\dagger}$, S.E. Maya-Rueda* and G. Mino-Aguilar* \\ *Autonomous University of Puebla (BUAP), Faculty of Electronics, Puebla, Mexico \\ Email: fermi.guerrero@correo.buap.mx

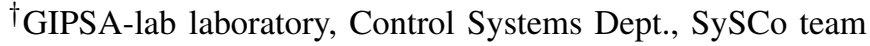 \\ CNRS-Univ. of Grenoble, ENSE3 BP 46, 38402 St Martin d'Hères Cedex, France, \\ Email: nicolas.marchand@gipsa-lab.inpg.fr \\ ${ }^{\ddagger}$ INSA Strasbourg and ICube, Strasbourg, France \\ Email: sylvain@durandchamontin.fr
}

\begin{abstract}
This paper presents the development of a collaborative event-based control applied to the problem of consensus and formation of a group of VTOL-UAVs (Vertical Take-off and Landing, Unmanned Aerial Vehicles) . Each VTOL-UAV decides, based on the difference of its current state (linear position and velocity) and its latest broadcast state, when it has to send a new value to its neighbors. The asymptotic convergence to average consensus or desired formation is depicted via numerical simulations.
\end{abstract}

\section{INTRODUCTION}

Motivated by applications in physics, biology and engineering the study of consensus of collections of agents (or dynamic systems) has become an important topic in control theory. Roughly speaking, consensus means to reach an agreement regarding a certain quantity of interest that depends on the state of all agents. A consensus algorithm (or protocol) is an interaction rule that specifies the information exchange between an agent and all of its neighbors on the network. Consensus problems have a long history in computer science and form the foundation of the field of distributed computing [1]. Distributed computation over networks has a tradition in systems and control theory e.g. [2] and [3].

Cooperative distributed control strategies for multiple vehicles have gained increased attention in recent years in the control community, owing to the fact that such strategies provide attractive solutions to large-scale multi-agent problems, both in terms of complexity in the formulation of the problem, as well as in terms of the computational load required for its solution. As an important branch of cooperative control, distributed cooperative attitude control for multiple rigid bodies has received much research attention. The motivation to consider rigid body dynamics comes from the fact that rigid body describes a very large class of physical systems of practical interest, e.g. spacecraft, UAVs, robot manipulators and wearable robots [4], [5].

An important aspect in the implementation of distributed algorithms is the communication and controller actuation schemes. With the development of embedded, miniaturized and interconnected systems, there is a growing interest in Networked Control Systems (NCSs) where the control loop is closed over a communication link [6], [7]. A network has several advantages, like flexibility in the configuration of the communication structure and the number of interconnected systems. However, it also has a considerable impact on the performance, notably because of communication delays and packet losses which avoid real-time control constraints to be meet and can even cause the instability of the control loop.

Recently, published works addressed resource aware implementations of the control law using event-based sampling, where the control law is event-driven. Such a paradigm calls for resources whenever they are indeed necessary, that is for instance when the dynamics of the controlled system varies, i.e. when some events occur. The development of event-based control strategies have their origins in the seminal works [8] and [9] where the the first event driven PID was developed. Event-based control usually relies on a triggering algorithm which takes the form of an event function $e: \mathcal{X} \rightarrow \mathbb{R}$ that indicates if one needs $(e \leq 0)$ or not $(e>0)$ to update the control value. $\mathcal{X}$ in general represents the state space. The basic event-based control strategies consist in level-crossing approaches like in [10] where $\mathcal{X}$ represents the output system. Other level-crossing approaches were reported in [11][12]. In more sophisticate approaches, the function $e$ takes the current state $x$ as input and a memory $m$ of $x$ last time $e$ became negative as in [13], [20]. Recent advances in event-triggered control can be classified into two main categories, Periodic Event-triggered Control scheme (PETC) and Continuous Event-triggered Control scheme (CETC). For PETC, a periodic sampling is given and the event function indicates the control must be updated at the next sampling instant [14] whereas in the Continuous Event-triggered Control scheme (CETC), the control function is updated instantaneously after the zeroing of the event function [15], [16], [17], [13].

In the context of cooperative distributed control, the event-based paradigm appears as a mean to reduce the communication bandwidth in the network since, contrary to the classical scheme, an event-based control invokes a communication between the different agents only when a certain condition is satisfied. In [18] an event-based implementation of the consensus protocol is presented. In the aforementioned work all agents continuously monitor their neighbors states, then each agent updates its control law not only at its own event-times, but also whenever one of its neighbors triggers an event. Later in [19] these disadvantages are addressed, and the authors developed an 
event-based control strategy for multi-agent average consensus where measurement broadcasts are scheduled in an event-based fashion, such that continuous monitoring of the neighbors states is no longer required.

Although the aforementioned approaches have shown benefits, these works were developed in a continuous event-triggered context, which means that the event-triggered condition has to be monitored continuously and it is necessary to guarantee non zero inter-execution time (Zeno behavior).

The control strategy proposed in the present paper is more in the spirit of the one proposed in [19]. However, the main difference is that the event-triggered condition is verified only periodically, and at every sampling period it is decided whether or not to broadcast a new state value to its neighbors, such that all agents states converge to the average of their initial conditions. With the proposed approach a minimal sampling period is guaranteed ( the control can not be updated more often than the a priori given sample period ) i.e., no Zeno behavior is guaranteed.

The proposed control technique is applied to the problem of consensus and formation of a group of VTOL-UAVs. Hence, each VTOL-UAV decides, based on the difference of its current state (linear position and velocity) and its latest broadcast state, when it has to send a new value to its neighbors. The asymptotic convergence to average consensus is guaranteed under such an event-triggered strategy. Numerical simulation are performed for the consensus of four quadrotors, where the effectiveness is illustrated via the comparison to traditional time-scheduled control. Besides the development of a collaborative event-triggered control, the hoped-for contribution of this paper is that of unification, so that the reader can see how mixing topics such as Unmanned Aerial Vehicles, nonlinear attitude control, and event-triggered collaborative control.

The paper is structured as follows. Section II contains mathematical preliminaires. In section III, the attitude control is given and an event-based strategy for position and velocity consensus is presented. Section IV is devoted to simulation results, which show the effectiveness of the proposed algorithm. Finally, in section V some conclusions are presented.

\section{PRELIMINARIES}

\section{A. Graph theory}

Consider $\mathcal{G}=\{\mathcal{V}, \mathcal{E}\}$ consisting of a set of vertices (or nodes) $\mathcal{V}=1, \ldots, N$ and edges $\mathcal{E}$. If there is an edge $(i, j)$ between nodes $i$ and $j$, then $i$ and $j$ are called adjacent, i.e. $\mathcal{E}=(i, j) \in \mathcal{V} \times \mathcal{V}: i, j$ adjacent. $\mathcal{G}$ is called undirected if $(i, j) \in \mathcal{E} \Leftrightarrow(i, j) \in \mathcal{E}$. The adjacency matrix $A$ is defined by $a_{i j}=1$ if $i$ and $j$ are adjacent and $a_{i j}=0$ otherwise. A path from $i$ to $j$ is a sequence of distinct nodes, starting from $i$ and ending with $j$, such that each pair of consecutive nodes is adjacent. If there is a path from $i$ to $j$, then $i$ and $j$ are called connected. if all pairs of nodes in $\mathcal{G}$ are connected, then $\mathcal{G}$ is called connected. The distance $d(i, j)$ between two nodes is the number of edges of the shortest path from $i$ to $j$. The diameter $d$ of $\mathcal{G}$ is the maximum distance $d(i, j)$ over all pairs of nodes. The degree matrix $D$ of $\mathcal{G}$ is the diagonal matrix with elements $d_{i}$ equal to the cardinality of node i's neighbor set $N_{i}=\{j \in \mathcal{V}:(i, j) \in \mathcal{E}$. The Laplacian matrix $\mathcal{L}$ of $\mathcal{G}$ is defined as $\mathcal{L}=D-A$. For undirected graphs, $\mathcal{L}$ is symmetric and positive semi-definite, i.e., $\mathcal{L}=\mathcal{L}^{T} \geq 0$. The row sums of $\mathcal{L}$ are zero. Thus, the vector of ones 1 is an eigenvector corresponding to eigenvalue $\lambda_{i}(\mathcal{G})=0$, i.e., $\mathcal{L} 1=0$. For connected graphs, $\mathcal{L}$ has exactly one zero eigenvalue, and the eigenvalues can be listed in increasing order $0=\lambda_{1}(\mathcal{G})<$ $\lambda_{2}(\mathcal{G}) \leq \ldots \leq \lambda_{N}(\mathcal{G})$. The second eigenvalue $\lambda_{2}(\mathcal{G})$ is call the algebraic connectivity.

\section{B. VTOL-UAVs model}

Firstly, assume that a VTOL-UAV can be modeled as a rigid body (see Fig. 1). Then, consider two orthogonal right-handed coordinate frames: the body coordinate frame, $\mathbf{E}^{b}=\left[\vec{e}_{1}^{b}, \vec{e}_{2}^{b}, \vec{e}_{3}^{b}\right]$, located at the center of mass of the rigid body and the inertial coordinate frame, $\mathbf{E}^{f}=\left[\vec{e}_{1}^{f}, \vec{e}_{2}{ }^{f}, \vec{e}_{3}{ }^{f}\right]$, located at some point in the space. The rotation of the body frame $\mathbf{E}^{b}$ with respect to the fixed frame $\mathbf{E}^{f}$ is represented by the attitude matrix $R \in S O(3)=\left\{R \in \mathbb{R}^{3 \times 3}: R^{T} R=\right.$ $I, \operatorname{det} R=1\}$.

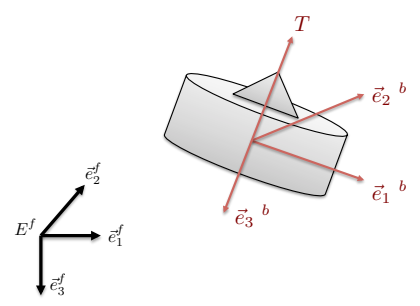

Fig. 1. Body-fixed and inertial reference frame

The cross product between two vectors $\xi, \chi \in \mathbb{R}^{3}$ is represented by a matrix multiplication $\left[\xi^{\times}\right] \chi=\xi \times \chi$, where $\left[\xi^{\times}\right]$is the well known skew-symmetric matrix.

The $n$-dimensional unit sphere embedded in $\mathbb{R}^{n+1}$ is denoted as $\mathbb{S}^{n}=\left\{x \in \mathbb{R}^{n+1}: x^{T} x=1\right\}$. Members of $S O(3)$ are often parametrized in terms of a rotation $\beta \in \mathbb{R}$ about a fixed axis $e_{v} \in \mathbb{S}^{2}$ by the map $\mathcal{U}: \mathbb{R} \times \mathbb{S}^{2} \rightarrow S O(3)$ defined as

$$
\mathcal{U}\left(\beta, e_{v}\right):=I_{3}+\sin (\beta)\left[e_{v}^{\times}\right]+(1-\cos (\beta))\left[e_{v}^{\times}\right]^{2}
$$

Hence, a unit quaternion, $q \in \mathbb{S}^{3}$, is defined as

$$
q:=\left(\begin{array}{c}
\cos \frac{\beta}{2} \\
e_{v} \sin \frac{\beta}{2}
\end{array}\right)=\left(\begin{array}{c}
q_{0} \\
q_{v}
\end{array}\right) \in \mathbb{S}^{3}
$$

$q_{v}=\left(\begin{array}{lll}q_{1} & q_{2} & q_{3}\end{array}\right)^{T} \in \mathbb{R}^{3}$ and $q_{0} \in \mathbb{R}$ are known as the vector and scalar parts of the quaternion respectively. $q$ represents an element of $S O(3)$ through the map $\mathcal{R}: \mathbb{S}^{3} \rightarrow S O(3)$ defined as

$$
\mathcal{R}:=I_{3}+2 q_{0}\left[q_{v}^{\times}\right]+2\left[q_{v}^{\times}\right]^{2}
$$

Note that $R=\mathcal{R}(q)=\mathcal{R}(-q)$ for each $q \in \mathbb{S}^{3}$, i.e. quaternions $q$ and $-q$ represent the same physical attitude.

Denoting by $\omega=\left(\begin{array}{lll}\omega_{1} & \omega_{2} & \omega_{3}\end{array}\right)^{T}$ the angular velocity vector of the body coordinate frame, $\mathbf{E}^{b}$ relative to the inertial coordinate frame, $\mathbf{E}^{f}$, expressed in $\mathbf{E}^{b}$, the kinematics equation is given by

$$
\left(\begin{array}{c}
\dot{q}_{0} \\
\dot{q}_{v}
\end{array}\right)=\frac{1}{2}\left(\begin{array}{c}
-q_{v}^{T} \\
I_{3} q_{0}+\left[q_{v}^{\times}\right]
\end{array}\right) \omega=\frac{1}{2} \Xi(q) \omega
$$

The attitude error is used to quantify the mismatch between two attitudes. If $q$ defines the current attitude quaternion and 
$q_{d}$ is the desired quaternion, i.e. the desired orientation, then the quaternion that represents the attitude error between the current orientation and the desired one is given by

$$
\tilde{q}=q_{d}^{-1} \otimes q=\left(\begin{array}{ll}
\tilde{q}_{0} & \tilde{q}_{v}^{T}
\end{array}\right)^{T}
$$

where $q^{-1}$ is the complementary rotation of the quaternion $q$ which is given by $q^{-1}=\left(\begin{array}{ll}q_{0} & -q_{v}^{T}\end{array}\right)^{T}$ and $\otimes$ denotes the quaternion multiplication [22]. In the case that the current quaternion and the desired one coincide, the quaternion error becomes $\tilde{q}=\left( \pm 10^{T}\right)^{T}$.

As it was mentioned before, the quaternion representation is redundant. As a consequence, the error mathematical model has two equilibrium and this fact must be considered in the stability analysis [23].

Now, consider a group of $N$-VTOL UAVs modeled as rigid bodies (see Fig. 2). Then according to the aforementioned and to [21], the six degrees of freedom model (position and attitude) of the system can be separated into translational and rotational motions, represented respectively by $\Sigma_{T_{i}}$ and $\Sigma_{R_{i}}$ in equation (6) and (7).

$$
\begin{gathered}
\Sigma_{T_{i}}:\left\{\begin{array}{l}
\dot{p}_{i}=v_{i} \\
\dot{v}_{i}=g \vec{e}_{3}^{f}-\frac{1}{m_{h_{i}}} R_{i}^{T} T_{i} \vec{e}_{3_{i}}^{b}
\end{array}\right. \\
\Sigma_{R_{i}}:\left\{\begin{array}{c}
\dot{q}_{i}=\frac{1}{2} \Xi\left(q_{i}\right) \omega_{i} \\
J_{i} \dot{\omega}_{i}=-\left[\omega_{i}^{\times}\right] J \omega_{i}+\Gamma_{i}
\end{array}\right.
\end{gathered}
$$

with $i \in \mathcal{N}=\{1, \ldots, N\} . m_{h_{i}}$ denotes the mass of the $i$ th VTOL-UAV and $J_{i}$ its inertial matrix expressed in $\mathbf{E}_{i}^{b} \cdot g$ is the gravity acceleration and $\vec{e}_{3_{i}}^{b}=\vec{e}_{3}^{f}=\left(\begin{array}{lll}0 & 0 & 1\end{array}\right)^{T} \cdot p_{i} \in \mathbb{R}^{3}$ represents the position of the aircraft's center of gravity, which coincides with the origin of frame $\mathbf{E}_{i}^{b}$, with respect to frame $\mathbf{E}^{f}, v_{i} \in \mathbb{R}^{3}$ its linear velocity in $\mathbf{E}^{f}$, and $\omega_{i} \in \mathbb{R}^{3}$ denotes the angular velocity expressed in $\mathbf{E}_{i}^{b} . \Gamma_{i} \in \mathbb{R}^{3}$ depends on the couples generated by the actuators, aerodynamic couples and external couples (environmental forces). In this paper, it is assumed that these torques are only generated by the actuators. $-T_{i} \vec{e}_{3_{i}}^{b}$ is the total thrust, expressed in $\mathbf{E}_{i}^{b}$.

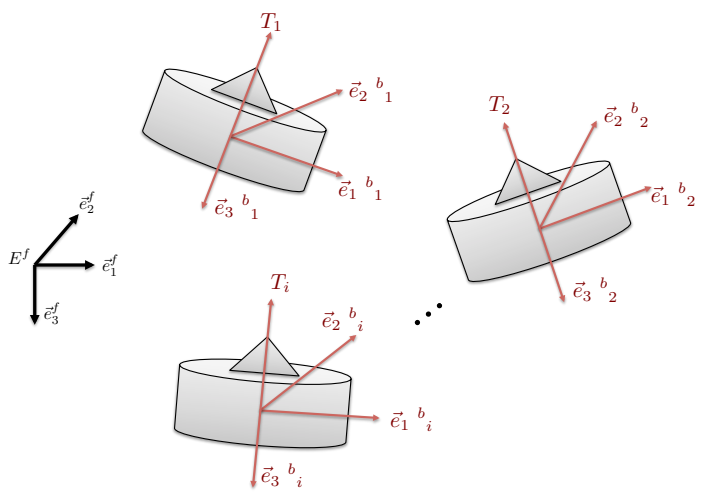

Fig. 2. Group of $N$-VTOL UAVs

\section{ATtiTUdE AND POSITION CONTROL}

In this subsection, a control law that stabilizes the system described by (6) and (7) is proposed.

Definition 3.1: Given a positive constant $M$, a continuous, nondecreasing function $\sigma_{M}: \mathbb{R} \rightarrow \mathbb{R}$ is defined by

$$
\begin{aligned}
& (1) \sigma_{M}(s)=s \text { if }|s|<M \\
& (2) \sigma_{M}(s)=\operatorname{sign}(s) M \text { elsewhere; }
\end{aligned}
$$

Then, one has the following result reported previously in [21] for the attitude stabilization of rigid bodies.

Theorem 3.2: Consider the $i$ th rigid body rotational dynamics described by (7) and the attitude error defined in (5) with the following bounded control inputs $\Gamma_{i}=\left(\Gamma_{i}^{1} \Gamma_{i}^{2} \Gamma_{i}^{3}\right)^{T}$ such that

$$
\Gamma_{i}^{l}=-\sigma_{M_{i}^{l}}\left(\frac{\kappa_{i} \omega_{i}^{l}}{\rho_{i}^{l}}+\kappa_{i} \tilde{q}_{v_{i}}^{l}\right)
$$

where $i \in \mathcal{N}=\{1, \ldots, N\} . \sigma_{M_{i}^{l}}(\cdot)$ with $l \in\{1,2,3\}$ are saturation functions as defined above. $M_{i}^{l}$ represents the physical bound on the $j$ th torque of the $i$ th rigid body. $\kappa_{i}$ is a real parameter such that $0<\kappa_{i} \leq \min _{l} M_{i}^{l} / 2 . \rho_{i}^{l}$ are strictly positive real parameters. Then the inputs (9) asymptotically stabilize the rigid body to the desired attitude $q_{d_{i}}$ (i.e. $\tilde{q}_{0_{i}}=$ $1, \tilde{q}_{v_{i}}=0, \omega_{i}=0$ ) with a domain of attraction for the attitude error and angular velocity equal to $\mathbb{S}^{3} \times \mathbb{R}^{3} \backslash\left(\begin{array}{lll}-1 & 0^{T} & 0^{T}\end{array}\right)^{T}$.

Proof: The proof follows the one presented in [21].

Now, the objective is to design a control law which stabilizes a group of VTOL-UAV to a certain position in the space (consensus problem), having the attitude stabilization problem solved. Consider (6) and (7). Note that the rotation matrix $R_{i}$ can be parameterized in function of Euler angles, through the map $\mathcal{R}(\phi, \theta, \psi): \mathbb{R}^{3} \rightarrow S O(3)$

$$
\begin{gathered}
R=\mathcal{R}(\phi, \theta, \psi)= \\
\left(\begin{array}{ccc}
\mathrm{C} \psi \mathrm{C} \theta & \mathrm{S} \psi \mathrm{C} \theta & -\mathrm{S} \theta \\
\mathrm{C} \psi \mathrm{S} \theta \mathrm{S} \phi-\mathrm{S} \psi \mathrm{C} \theta & \mathrm{S} \phi \mathrm{S} \theta \mathrm{S} \psi+\mathrm{C} \psi \mathrm{C} \phi & \mathrm{C} \theta \mathrm{S} \phi \\
\mathrm{C} \psi \mathrm{C} \phi \mathrm{S} \theta+\mathrm{S} \psi \mathrm{S} \phi & \mathrm{S} \theta \mathrm{S} \psi \mathrm{C} \phi-\mathrm{C} \psi \mathrm{S} \phi & \mathrm{C} \theta \mathrm{C} \phi
\end{array}\right),
\end{gathered}
$$

Assume that using the control law (9), one can stabilize the yaw dynamics of $i$ th VTOL, that is $\psi_{i}=0$. Then, after a sufficiently long time, system (6) becomes:

$$
\begin{aligned}
& \left(\begin{array}{c}
\dot{p}_{x_{i}} \\
\dot{p}_{y_{i}} \\
\dot{p}_{z_{i}}
\end{array}\right)=\left(\begin{array}{c}
v_{x_{i}} \\
v_{y_{i}} \\
v_{z_{i}}
\end{array}\right) \\
& \left(\begin{array}{c}
\dot{v}_{x_{i}} \\
\dot{v}_{y_{i}} \\
\dot{v}_{z_{i}}
\end{array}\right)=\left(\begin{array}{c}
-\frac{T_{i}}{m_{i}} \operatorname{sen} \theta_{i} \\
\frac{T_{i}}{m_{i}} \operatorname{sen} \phi_{i} \cos \theta_{i} \\
\frac{T_{i}}{m_{i}} \cos \phi_{i} \cos \theta_{i}-g
\end{array}\right)
\end{aligned}
$$

$\theta_{i}$ and $\phi_{i}$ can be viewed as an intermediate input to control (11)-(12). With an appropriate choice of these target configuration as proposed in [24], [25], it will be possible to 
transform (11)-(12) into three double integrators. For this, let us define

$$
\begin{aligned}
\phi_{d_{i}} & :=\arctan \left(\frac{r_{y_{i}}}{r_{z_{i}}+g}\right), \\
\theta_{d_{i}} & :=\arcsin \left(\frac{-r_{x_{i}}}{\sqrt{r_{x_{i}}^{2}+r_{y_{i}}^{2}+\left(r_{z_{i}}+g\right)^{2}}}\right)
\end{aligned}
$$

where $r_{x_{i}}, r_{y_{i}}$ and $r_{z_{i}}$ will be defined after. Then, choose as positive thrust the input control

$$
T_{i}=m_{i} \sqrt{r_{x_{i}}^{2}+r_{y_{i}}^{2}+\left(r_{z_{i}}+g\right)^{2}}
$$

By taking (13)-(14), it follows

$$
\begin{aligned}
& \Sigma_{x_{i}}:=\left\{\begin{array}{l}
\dot{p}_{x_{i}}=v_{x_{i}} \\
\dot{v}_{x_{i}}=r_{x_{i}}
\end{array}\right. \\
& \Sigma_{y_{i}}:=\left\{\begin{array}{l}
\dot{p}_{y_{i}}=v_{y_{i}} \\
\dot{v}_{y_{i}}=r_{y_{i}}
\end{array}\right. \\
& \Sigma_{z_{i}}:=\left\{\begin{array}{l}
\dot{p}_{z_{i}}=v_{z_{i}} \\
\dot{v}_{z_{i}}=r_{z_{i}}
\end{array}\right.
\end{aligned}
$$

Now the aim is to adapt and apply a control law and a triggering rule, previously developed in [19], in order to determine, based on local information, when the $i$ th VTOL-UAV (agent) has to trigger and broadcast a new state value to its neighbors, such that all agents' states converge to the average of their initial conditions. Each agent consists of a position controller as show in Fig. 3 In this case the position controller of system $i$ monitors its own state $\xi_{i}=\left(p_{i}^{T} v_{i}^{T}\right)^{T}$ continuously. Based on local information, it decides when to broadcast its current state over the network. The latest broadcast state of system $i$ given by $\hat{\xi}_{i}=\xi_{i}\left(t_{k}^{i}\right), t \in\left[t_{k}^{i}, t_{k+1}^{i}[\right.$, where $t_{0}^{i}, t_{1}^{i}, t_{2}^{i} \ldots$ is the sequence of event times of agent $i$.

Hence a simple consensus algorithm to reach an agreement regarding the state of $N$ VTOL-UAVs can be expressed:

$$
\begin{aligned}
& r_{x_{i}}=\sum_{j \in N_{i}}\left(\hat{p}_{x_{i}}-\hat{p}_{x_{j}}\right)-\mu \sum_{j \in N_{i}}\left(\hat{v}_{x_{i}}-\hat{v}_{x_{j}}\right) \\
& r_{y_{i}}=\sum_{j \in N_{i}}\left(\hat{p}_{y_{i}}-\hat{p}_{y_{j}}\right)-\mu \sum_{j \in N_{i}}\left(\hat{v}_{y_{i}}-\hat{v}_{y_{j}}\right) \\
& r_{z_{i}}=\sum_{j \in N_{i}}\left(\hat{p}_{z_{i}}-\hat{p}_{z_{j}}\right)-\mu \sum_{j \in N_{i}}\left(\hat{v}_{z_{i}}-\hat{v}_{z_{j}}\right)
\end{aligned}
$$

with $\mu>0$. Let us define the stack vectors $p_{x}(t)=$ $\left(p_{x_{1}}(t) \ldots p_{x_{N}}(t)\right)^{T}, \quad p_{y}(t)=\left(p_{y_{1}}(t) \ldots p_{y_{N}}(t)\right)^{T}, p_{z}(t)=$ $\left(p_{z_{1}}(t) \ldots p_{z_{N}}(t)\right)^{T}, \quad v_{x}(t)=\left(v_{x_{1}}(t) \ldots v_{x_{N}}(t)\right)^{T}, v_{y}(t)=$ $\left(v_{y_{1}}(t) \ldots v_{y_{N}}(t)\right)^{T}, v_{z}(t)=\left(v_{z_{1}}(t) \ldots v_{z_{N}}(t)\right)^{T}$, and $\hat{p}_{x}(t)=$ $\left(\hat{p}_{x_{1}}(t) \ldots \hat{p}_{x_{N}}(t)\right)^{T}, \quad \hat{p}_{y}(t)=\left(\hat{p}_{y_{1}}(t) \ldots \hat{p}_{y_{N}}(t)\right)^{T}, \hat{p}_{z}(t)=$ $\left(\hat{p}_{z_{1}}(t) \ldots \hat{p}_{z_{N}}(t)\right)^{T}, \hat{v}_{x}(t)=\left(\hat{v}_{x_{1}}(t) \ldots \hat{v}_{x_{N}}(t)\right)^{T}, \hat{v}_{y}(t)=$ $\left(\hat{v}_{y_{1}}(t) \ldots \hat{v}_{y_{N}}(t)\right)^{T}, \hat{v}_{z}(t)=\left(\hat{v}_{z_{1}}(t) \ldots \hat{v}_{z_{N}}(t)\right)^{T}$ Furthermore, let us define the following measurement errors for the linear position and velocity

$$
\begin{aligned}
e_{p_{x}, i}(t) & =\hat{p}_{x_{i}}(t)-p_{x_{i}}(t) \\
e_{p_{y}, i}(t) & =\hat{p}_{y_{i}}(t)-p_{y_{i}}(t) \\
e_{p_{z}, i}(t) & =\hat{p}_{z_{i}}(t)-p_{z_{i}}(t) \\
e_{p, i}(t) & =\left(e_{p_{x}, i}^{T} e_{p_{y}, i}^{T} e_{p_{z}, i}^{T}\right)^{T}
\end{aligned}
$$

$$
\begin{aligned}
e_{v_{x}, i}(t) & =\hat{v}_{x_{i}}(t)-v_{x_{i}}(t) \\
e_{v_{y}, i}(t) & =\hat{v}_{y_{i}}(t)-v_{y_{i}}(t) \\
e_{v_{z}, i}(t) & =\hat{v}_{z_{i}}(t)-v_{z_{i}}(t) \\
e_{v, i}(t) & =\left(e_{v_{x}, i}^{T} e_{v_{y}, i}^{T} e_{v_{z}, i}^{T}\right)^{T}
\end{aligned}
$$

Hence, the control law (18) can be rewritten as

$$
\begin{aligned}
& r_{x}=-L\left(p_{x}(t)+\mu v_{x}(t)+e_{p_{x}}(t)+\mu e_{v_{x}}(t)\right) \\
& r_{y}=-L\left(p_{y}(t)+\mu v_{y}(t)+e_{p_{y}}(t)+\mu e_{y_{x}}(t)\right) \\
& r_{z}=-L\left(p_{z}(t)+\mu v_{z}(t)+e_{p_{z}}(t)+\mu e_{z_{x}}(t)\right)
\end{aligned}
$$

where $e_{p_{x}}(t), e_{p_{y}}(t), e_{p_{z}}(t), e_{v_{x}}(t), e_{v_{y}}(t), e_{v_{z}}(t)$ are the measurement errors stack vectors. Then, the closed-loop system becomes

$$
\left(\begin{array}{c}
\dot{p}_{x} \\
\dot{v}_{x}
\end{array}\right)=\Upsilon\left(\begin{array}{l}
p_{x} \\
v_{x}
\end{array}\right)-\left(\begin{array}{ll}
0 & 0 \\
\mathcal{L} & \mathcal{L}
\end{array}\right) e_{x}(t)
$$

where

$$
\Upsilon=\left(\begin{array}{cc}
0 & I \\
-\mathcal{L} & -\mu \mathcal{L}
\end{array}\right)
$$

and

$$
e_{x}(t)=\left(\begin{array}{c}
e_{p_{x}}(t) \\
\mu e_{v_{x}}(t)
\end{array}\right)
$$

a similar procedure can be done for $y$ and $z$ coordinates. Define the average velocity $b_{(x, y, z)}$ and the average position $a_{(x, y, z)}+$ $b_{(x, y, z)} t$ of all the agents, with

$$
\begin{aligned}
& a_{x}=\frac{1}{N} \sum_{i \in \mathcal{V}} p_{x_{i}}(0), a_{y}=\frac{1}{N} \sum_{i \in \mathcal{V}} p_{y_{i}}(0), a_{z}=\frac{1}{N} \sum_{i \in \mathcal{V}} p_{z_{i}}(0) \\
& b_{x}=\frac{1}{N} \sum_{i \in \mathcal{V}} v_{x_{i}}(0), b_{y}=\frac{1}{N} \sum_{i \in \mathcal{V}} v_{y_{i}}(0), b_{z}=\frac{1}{N} \sum_{i \in \mathcal{V}} v_{z_{i}}(0)
\end{aligned}
$$

Then, on a has the following result

Corollary 3.3: Consider the group of VTOL-UAVs (11)-(12) with control laws (13)-(14) and (18). Suppose the trigger function is given by

$$
\begin{aligned}
f_{i}\left(t, e_{p, i}(t), e_{v, i}(t)\right) & =\left\|\left(e_{p, i}^{T}(t) \mu e_{v, i}^{T}(t)\right)^{T}\right\| \\
& -\left(c_{0}+c_{1} e^{-\alpha t}\right)
\end{aligned}
$$

with constants $c_{0}, c_{1} \geqslant 0$ and $c_{0}+c_{1}>0$, and $0<$ $\alpha<\left|\operatorname{Re}\left(\lambda_{3}(\Upsilon)\right)\right|$. Then, for all initial conditions $\xi_{i}(0)=$ $\left(p_{i}^{T}(0) v_{i}^{T}(0)\right)^{T}$ the average velocity and average position consensus are reached, with an error that depends on the value of $c_{0}$ of the trigger function, the eigenvalues of $\Upsilon$ and the number of agents in the graph [19].

Remark 3.4: Consensus algorithms can be extended to formation control if the formation is represented by vectors of relative positions of neighboring agents. Let us denote by 
$\Delta_{(\cdot)_{i j}}$ the desired constant offset between the $i$ th and the $j$ th vehicle. Then control law (18) can be modified as following

$$
\begin{aligned}
r_{x_{i}} & =\sum_{j \in N_{i}}\left(\hat{p}_{x_{i}}-\hat{p}_{x_{j}}-\Delta_{x_{i j}}\right)-\mu \sum_{j \in N_{i}}\left(\hat{v}_{x_{i}}-\hat{v}_{x_{j}}\right) \\
r_{y_{i}} & =\sum_{j \in N_{i}}\left(\hat{p}_{y_{i}}-\hat{p}_{y_{j}}-\Delta_{y_{i j}}\right)-\mu \sum_{j \in N_{i}}\left(\hat{v}_{y_{i}}-\hat{v}_{y_{j}}\right) \\
r_{z_{i}} & =\sum_{j \in N_{i}}\left(\hat{p}_{z_{i}}-\hat{p}_{z_{j}}-\Delta_{z_{i j}}\right)-\mu \sum_{j \in N_{i}}\left(\hat{v}_{z_{i}}-\hat{v}_{z_{j}}\right)
\end{aligned}
$$

The Fig.3 depict the control law running in the $i$ th vehicle.

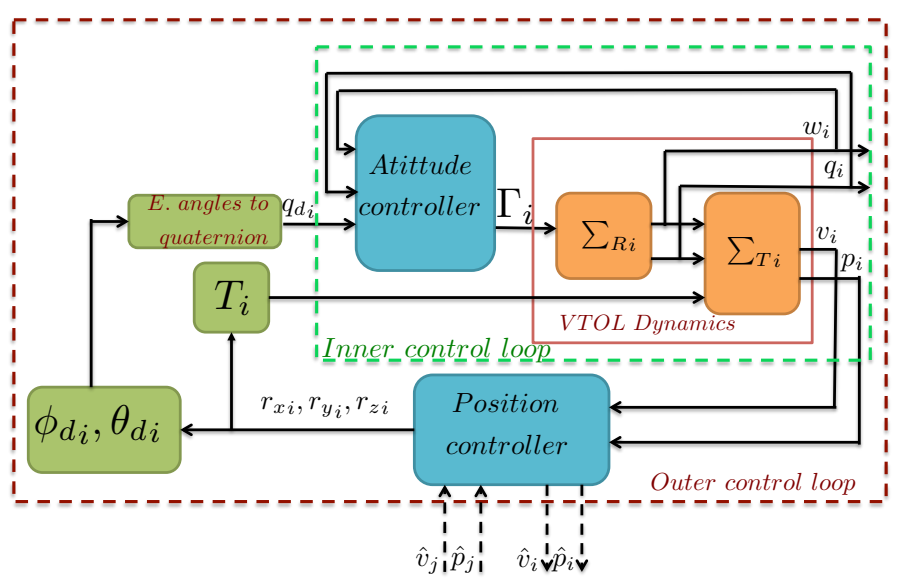

Fig. 3. Block diagram of the even-based control strategy

\section{Simulations Results}

In this section, the proposed control algorithms are verified and illustrated through simulations. The VTOL vehicle considered in the simulation is the well known four-rotor mini-helicopter so called quadrotor modeled as (6)-(7). Several simulations were carried out with a focus on two main outcomes: consensus and formation of VTOL vehicles.

\section{A. Quadrotor model}

The quadrotor is a small aerial vehicle that belongs to the VTOL (Vertical Taking Off and Landing) class of aircrafts. It is lifted and propelled, forward and laterally, by controlling the rotational speed of four blades mounted at the four ends of a simple cross and driven by four DC Brushless motors (BLDC). On such a platform (see Fig. 4), given that the front and rear motors rotate counter-clockwise while the other two rotate clockwise, gyroscopic effects and aerodynamic torques tend to cancel each other out in trimmed flight. The rotation of the four rotors generates a vertical force, called the thrust $T$, equal to the sum of the thrusts of each rotor $\left(T=f_{1}+f_{2}+f_{3}+f_{4}\right)$. The pitch movement $\theta$ is obtained by increasing/decreasing the speed of the rear motor while decreasing/increasing the speed of the front motor. The roll movement $\phi$ is obtained similarly using the lateral motors. The yaw movement $\psi$ is obtained by increasing/decreasing the speed of the front and rear motors while decreasing/increasing the speed of the lateral motors. In order to avoid any linear movement of the quadrotor, these maneuvers should be achieved while maintaining a value of the total thrust $T$ that balances the aircraft weight. In order to model the system's dynamics, two frames are defined: a fixed frame in the space $\mathbf{E}^{f}=\left[\vec{e}_{1}^{f}, \vec{e}_{2}^{f}, \vec{e}_{3}{ }^{f}\right]$ and a body-fixed frame $\mathbf{E}^{b}=\left[\vec{e}_{1}^{b}, \vec{e}_{2}^{b}, \vec{e}_{3}^{b}\right]$, attached to the quadrotor at its center of gravity, as shown in Fig. 4.

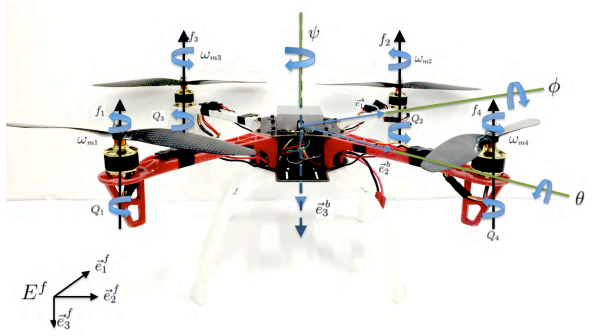

Fig. 4. Quadrotor: fixed frame $\mathbf{E}^{f}=\left[\vec{e}_{1}^{f}, \vec{e}_{2}^{f}, \vec{e}_{3}^{f}\right]$ and body-fixed frame $\mathbf{E}^{b}=\left[\vec{e}_{1}^{b}, \vec{e}_{2}^{b}, \vec{e}_{3}^{b}\right]$

The components of the control torque vector $\Gamma$ generated by the rotors are given by:

$$
\begin{aligned}
\Gamma_{1} & =d b_{m}\left(u_{m 3}-u_{m 4}\right) \\
\Gamma_{2} & =d b_{m}\left(u_{m 1}-u_{m 2}\right) \\
\Gamma_{3} & =k_{m}\left(-u_{m 1}+u_{m 2}-u_{m 3}+u_{m 4}\right) \\
T & =b_{m} \sum_{\bar{l}=1}^{4} u_{m \bar{l}}
\end{aligned}
$$

with $d$ being the distance from one rotor to the center of mass of the quadrotor. The specification and parameters of the quadrotor, used for the simulation, are given in the Table I.

\begin{tabular}{|c|c|c|c|}
\hline Parameter & Description & Value & Units \\
\hline$m$ & Mass & 1.490 & $\mathrm{Kg}$ \\
$d$ & Distance & 0.27 & $\mathrm{~m}$ \\
$J_{x}$ & Inertia in x-axis & $34.3 \times 10^{-3}$ & $\mathrm{Kg} \cdot \mathrm{m}^{2}$ \\
$J_{y}$ & Inertia in y-axis & $34.4 \times 10^{-3}$ & $\mathrm{Kg} \cdot \mathrm{m}^{2}$ \\
$J_{z}$ & Inertia in z-axis & $52.9 \times 10^{-3}$ & $\mathrm{Kg} \cdot \mathrm{m}^{2}$ \\
$b_{m}$ & Proportionality Constant & 8548 & $\mathrm{~N} / \mathrm{s}$ \\
$k_{m}$ & Proportionality Constant & 1898 & $\mathrm{~N} \cdot \mathrm{m} / \mathrm{s}$ \\
\hline
\end{tabular}

For this simulation, the control law (9) is implemented with control gains: $\rho_{1,2}=4.2, \rho_{1,2}=1.74$ and $\kappa=0.075$.

\section{B. Consensus of VTOL vehicles}

For this propose, four agents are employed, each one represents a VTOL - UAV in simulation. The communication graph implemented in the collaborative system is illustrated in Fig. 5. This simulation was performed in a time stamp of 20 seconds. For the event function, $c_{0}=0.02$ and for simplicity $c_{1}=0$ was chosen. The sampling time is 0.015 seconds. In the following charts, each agent is represented with a different color. This color code will remain the same throughout the paper. The initial conditions for position and speed of each agent are shown in table II. 


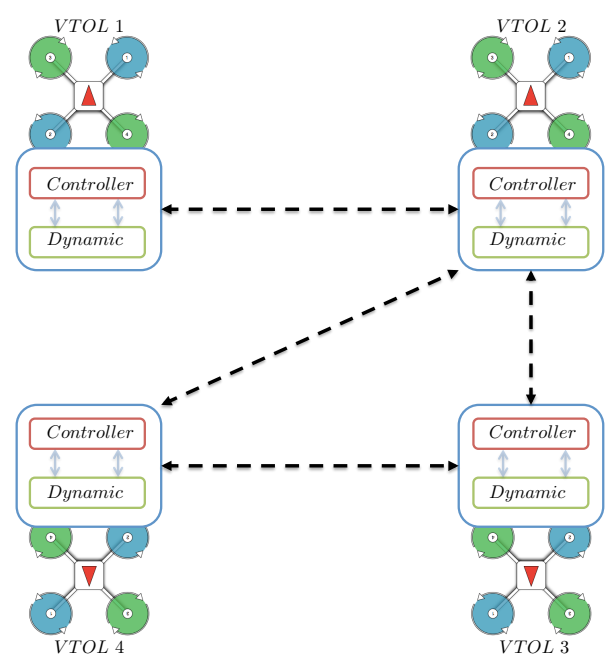

Fig. 5. Comunicacion graph $\mathcal{G}$

\begin{tabular}{||c|c|c||}
\hline \hline \multicolumn{4}{|c|}{ Initial conditions } \\
\hline Agent & I. C. Positions & I.C. Speeds \\
\hline VTOL 1 & {$\left[\begin{array}{lll}3 & 2 & -3\end{array}\right]$} & {$\left[\begin{array}{lll}0.2 & -0.3 & 0.02\end{array}\right]$} \\
\hline VTOL 2 & {$\left[\begin{array}{lll}2 & -3 & -6\end{array}\right]$} & {$\left[\begin{array}{lll}-0.2 & -0.03 & 0.2\end{array}\right]$} \\
\hline VTOL 3 & {$\left[\begin{array}{lll}4 & -2 & -1\end{array}\right]$} & {$\left[\begin{array}{lll}-0.3 & 0.1 & -0.04\end{array}\right]$} \\
\hline VTOL 4 & {$\left[\begin{array}{llll}-4 & -1 & 3\end{array}\right]$} & {$\left[\begin{array}{lll}0.3 & -0.1 & -0.4\end{array}\right]$} \\
\hline
\end{tabular}

Note that all agents start with different initial conditions during the simulation. The position evolution for the four vehicles is depicted in Fig. 6. One can see that the consensus is reached at 18 seconds. Furthermore, in Fig. 7 the velocities evolution are shown. Note that the four vehicles reach the same velocity in approximately 18 seconds too, i.e. they achieve consensus.
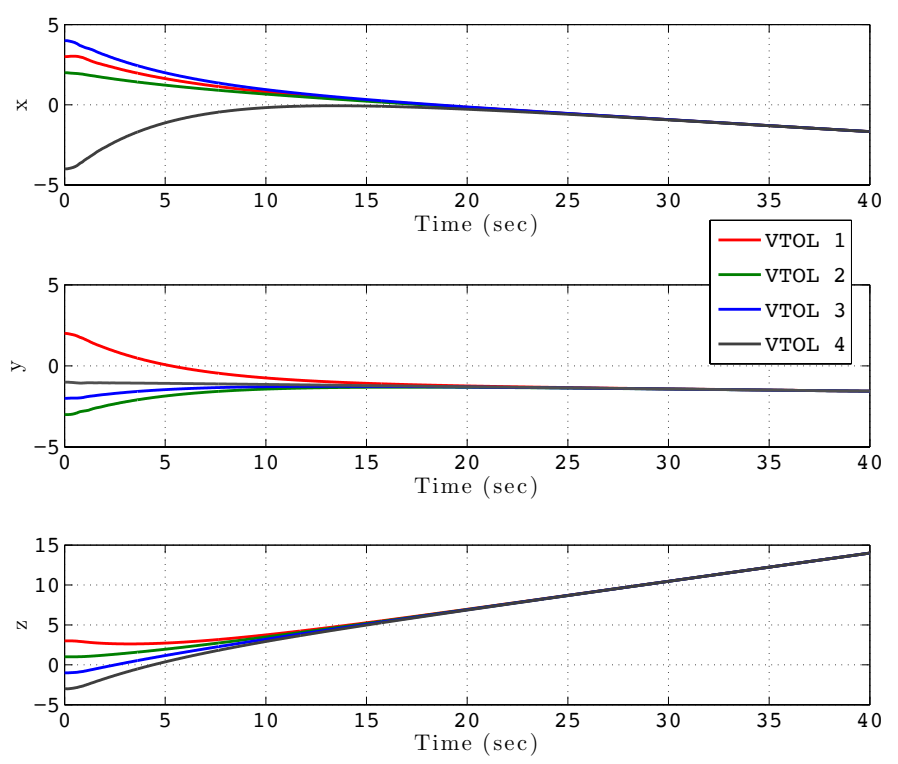

Fig. 6. Linear position vectors, $\left(p_{x_{i}}, p_{y_{i}}, p_{z_{i}}\right) \mathrm{m}$.

The difference between a continuous-time approach and an event-triggered approach to the problem of communications
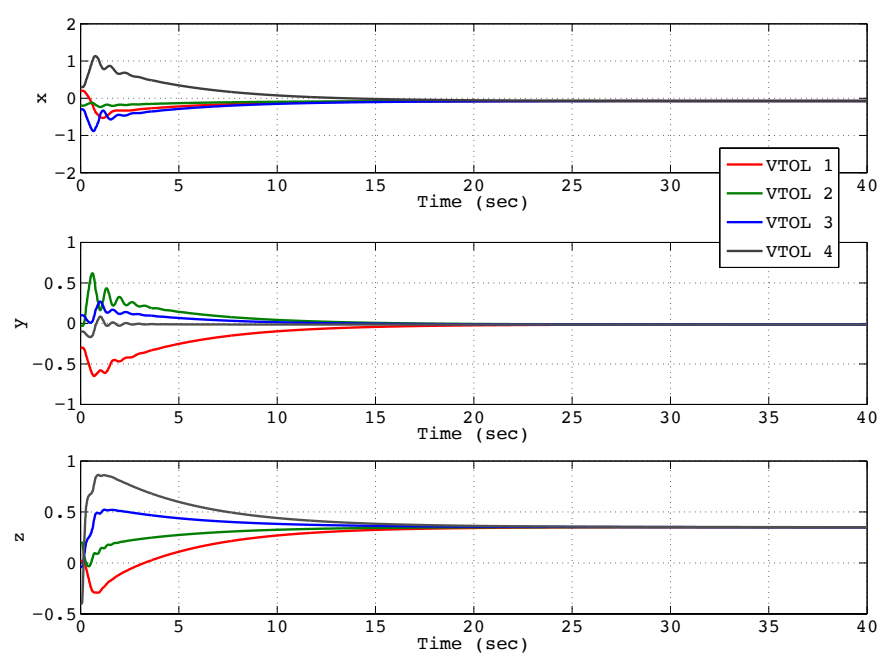

Fig. 7. Linear velocity vectors, $\left(v_{x_{i}}, v_{y_{i}}, v_{z_{i}}\right) \mathrm{m} / \mathrm{s}$.

in collaborative systems is highlighted in Fig. 8. Here, one observes the instant when the event occurs and the vehicle transmits its state to its immediate neighbors. During the first seconds, there exist many events. However, the number of events is dramatically reduced as the system reaches the consensus. The maximum number of events per agent was 279 (VTOL 4) while the minimum was 194 (VTOL 2). Table III presents the total number of events for each VTOL during simulation.

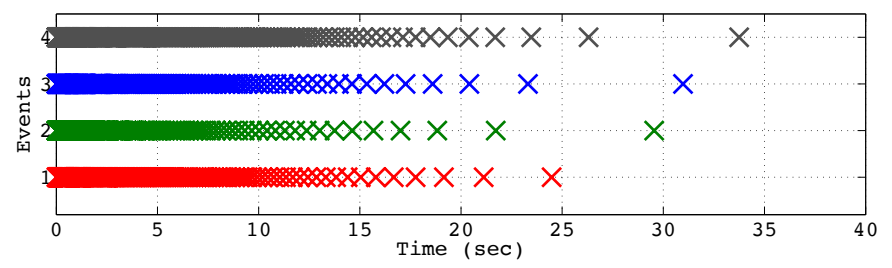

Fig. 8. Events occurrence

\begin{tabular}{||c|c|c||}
\hline \hline \multicolumn{3}{|c||}{ Consensus of VTOL } \\
\hline Agent & Sampling time & Times event-based control \\
\hline VTOL 1 & $0.015 \mathrm{sec}$ & 202 \\
\hline VTOL 2 & $0.015 \mathrm{sec}$ & 194 \\
\hline VTOL 3 & $0.015 \mathrm{sec}$ & 196 \\
\hline VTOL 4 & $0.015 \mathrm{sec}$ & 279 \\
\hline \multicolumn{2}{|c|}{ TABLE III. AMOUNT OF EVENTS } \\
\hline
\end{tabular}

\section{Formation of VTOL}

In this simulation, the control objective is to guarantee that the four vehicles maintain a pre-defined formation pattern, described by a square. The desired inter-vehicle relative position vector is $\Delta_{i j}=\left(\Delta_{i}-\Delta_{j}\right)$ with $\Delta_{1}=\left(\begin{array}{lll}3 & 3 & 0\end{array}\right)^{T}$, $\Delta_{2}=\left(\begin{array}{lll}-3 & 3 & 0\end{array}\right)^{T}, \Delta_{3}=\left(\begin{array}{lll}-3 & -3 & 0\end{array}\right)^{T}, \Delta_{4}=\left(\begin{array}{lll}3 & -3 & 0\end{array}\right)^{T}$. The information flow is the one of the Fig. 5 and the initial conditions of each vehicle are shown in table IV. The obtained results in this case are given in Figs. 9 and 10, which depict, respectively, the vehicle linear velocities and positions vectors in space. One observers from these figures that the control 
objective is achieved using the event-triggered strategy control. The events occurrences are shown in Fig. 11, which illustrates that during the first seconds there exist several occurrences and they are reduced while the vehicle reaches the formation.

\begin{tabular}{||c|c|c||}
\hline \hline \multicolumn{4}{|c|}{ Initial conditions } \\
\hline Agent & I. C. Positions & I.C. Speeds \\
\hline VTOL 1 & {$\left[\begin{array}{lll}3 & 2 & -3\end{array}\right]$} & {$\left[\begin{array}{llll}0.4 & 0.2 & -0.1\end{array}\right]$} \\
\hline VTOL 2 & {$\left[\begin{array}{lll}2 & -3 & -6\end{array}\right]$} & {$\left[\begin{array}{lll}0.2 & 0.3 & 0.1\end{array}\right]$} \\
\hline VTOL 3 & {$\left[\begin{array}{llll}4 & -2 & -1\end{array}\right]$} & {$\left[\begin{array}{lll}0.1 & 0.2 & 0.5\end{array}\right]$} \\
\hline VTOL 4 & {$\left[\begin{array}{lll}-4 & -1 & 3\end{array}\right]$} & {$\left[\begin{array}{lll}-0.4 & -0.1 & 0.2\end{array}\right]$} \\
\hline
\end{tabular}
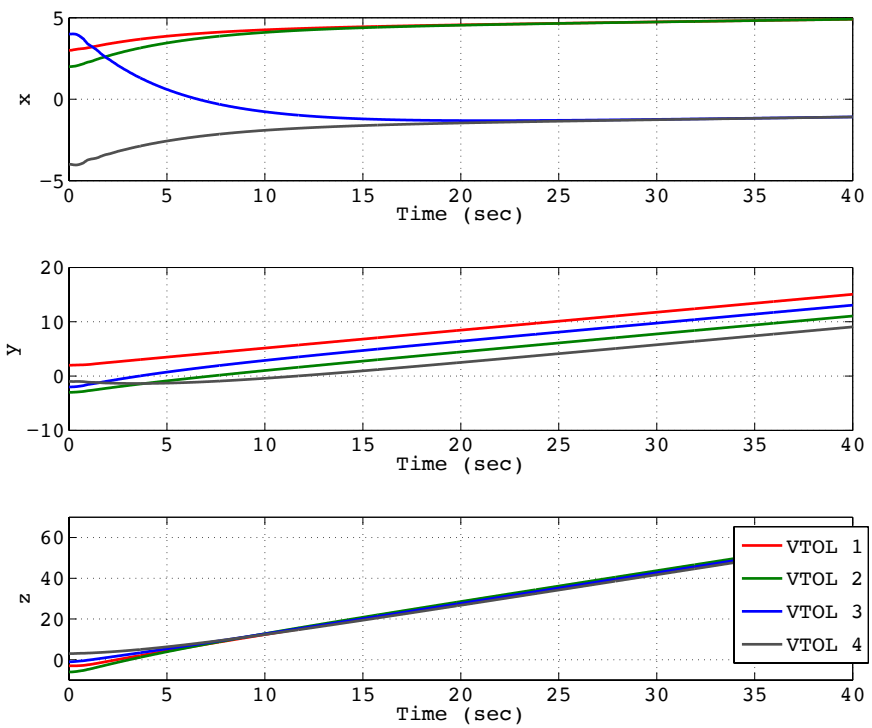

Fig. 9. Linear position vectors, $\left(p_{x_{i}}, p_{y_{i}}, p_{z_{i}}\right) \mathrm{m}$.
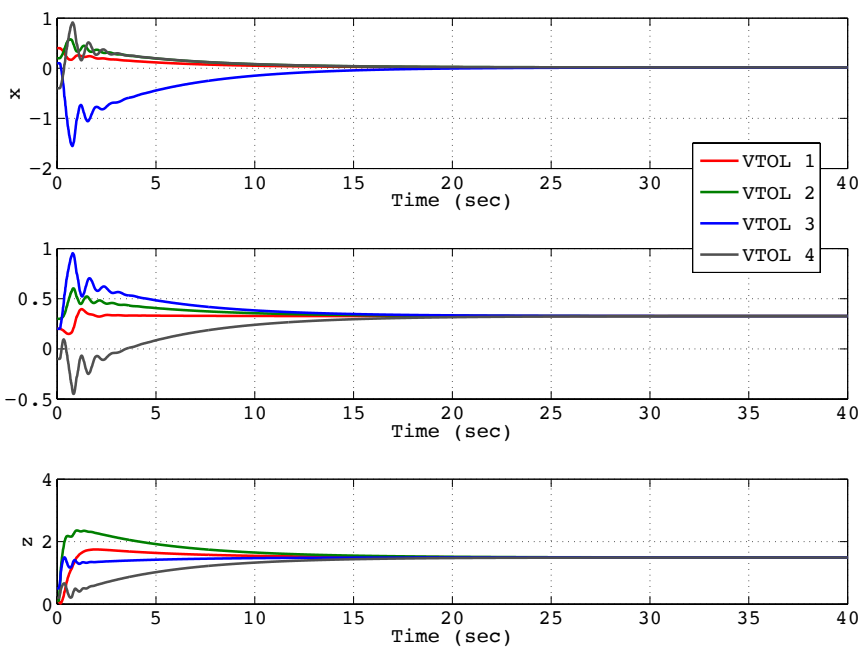

Fig. 10. Linear velocity vectors, $\left(v_{x_{i}}, v_{y_{i}}, v_{z_{i}}\right) \mathrm{m} / \mathrm{s}$.

In the classical frame (time-triggered control), the state should be broadcast 2666 times for a span of 40 seconds, since the sampling time is $0.015 \mathrm{sec}$. In the table $\mathrm{V}$ shows quantitatively the number of events occurred. In average,

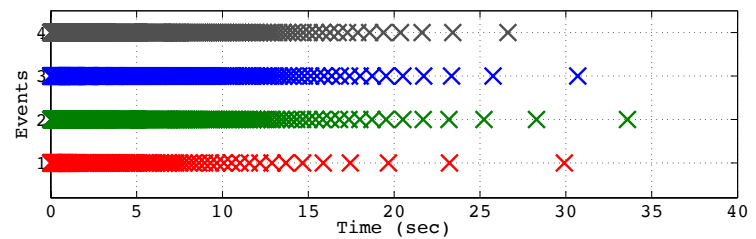

Fig. 11. Events occurrence

the state was transmitted to its immediate neighbors only 259 times, during the 40 seconds simulation time, which represents (in average) a reduction of $90.2 \%$. Finally, the Fig. 12 illustrates the vehicle position in space. In this figure the same color code is used to identify vehicles. The figure has three indicators, "x" denotes the initial positions with $t=0 \mathrm{sec}$, " + " indicates the position of the agents with $t=20 \mathrm{sec}$ and "o" marks the position of the same agents with $t=30 \mathrm{sec}$.

\begin{tabular}{||c|c|c||}
\hline \multicolumn{3}{|c|}{ Consensus of VTOL } \\
\hline Agent & Sampling time & Times event-based control \\
\hline VTOL 1 & $0.015 \mathrm{sec}$ & 150 \\
\hline VTOL 2 & $0.015 \mathrm{sec}$ & 274 \\
\hline VTOL 3 & $0.015 \mathrm{sec}$ & 299 \\
\hline VTOL 4 & $0.015 \mathrm{sec}$ & 314 \\
\hline
\end{tabular}

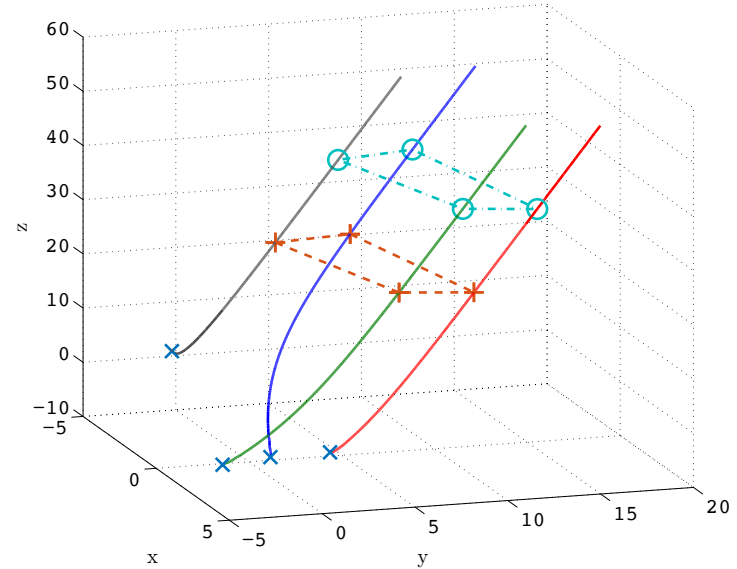

Fig. 12. Evolution of the position ("x" $t=0 \mathrm{sec}, "+" t=20 \mathrm{sec}$ and "o" $\mathrm{t}=30 \mathrm{sec})$

\section{Conclusion}

In this work, a colaborative control strategy applied the problem of consensus and formation of a group of VTOL is proposed. A collaborative control strategy is applied to the problem of consensus and formation flight of a VTOL group. The attitude control and consensus were tested and verified in simulation. Although the operation of the designed strategies is an important point of this work, we strongly believe that the greatest contribution is the development of an event-based control strategy used in the transmission of states 
between agents. This event-based control algorithm reduces the number of transmissions between agents approximately by $80 \%$, which demonstrate the superiority in terms of load on the communication medium.

Future work will address the real-time experimentation of the proposed strategy.

\section{REFERENCES}

[1] N. A. Lynch, Distributed algorithms. Morgan Kaufmann, 1996

[2] V. Borkar and P. P. Varaiya, "Asymptotic agreement in distributed estimation," Automatic Control, IEEE Transactions on, vol. 27, no. 3, pp. 650-655, 1982.

[3] J. N. Tsitsiklis, "Problems in decentralized decision making and computation.” DTIC Document, Tech. Rep., 1984.

[4] A. Abdessameud and A. Tayebi, "Attitude synchronization of a group of spacecraft without velocity measurements," Automatic Control, IEEE Transactions on, vol. 54, no. 11, pp. 2642-2648, 2009.

[5] W. Ren, "Formation keeping and attitude alignment for multiple spacecraft through local interactions," Journal of Guidance, Control, and Dynamics, vol. 30, no. 2, pp. 633-638, 2007.

[6] R. Olfati-Saber and J. S. Shamma, "Consensus filters for sensor networks and distributed sensor fusion," in Decision and Control, 2005 and 2005 European Control Conference. CDC-ECC'05. 44th IEEE Conference on. IEEE, 2005, pp. 6698-6703.

[7] G. S. Seyboth, D. V. Dimarogonas, and K. H. Johansson, "Control of multi-agent systems via event-based communication," in Proceedings of the 18th IFAC World Congress, 2011, 2011.

[8] K. J. Åström and B. Bernhardsson, "Comparison of riemann and lebesque sampling for first order stochastic systems," in Proceeding of the 41st IEEE Conference on Decision and Control, 2002, vol. 2. IEEE, 2002, pp. 2011-2016.

[9] K.-E. Årzén, "A simple event-based PID controller," in Preprints 14th World Congress of IFAC, Beijing, P.R. China, Jan. 1999.

[10] S. Durand and N. Marchand, "An event-based pid controller with low computational cost," in 8th International Conference on Sampling Theory and Applications (SampTA'09), 2009, pp. Special-session.

[11] M. Velasco, P. Martí, and E. Bini, "On lyapunov sampling for event-driven controllers," in Decision and Control, 2009 held jointly with the 2009 28th Chinese Control Conference. CDC/CCC 2009. Proceedings of the 48th IEEE Conference on. IEEE, 2009, pp. $6238-6243$.

[12] S. Durand, N. Marchand, and J. F. Guerrero Castellanos, "Simple Lyapunov Sampling for Event-Driven Control," in 18th IFAC World Congress (IFAC WC 2011), Milan, Italy, Aug. 2011, p. 7 p.

[13] N. Marchand, S. Durand, and J. F. Guerrero-Castellanos, "A general formula for event-based stabilization of nonlinear systems," Automatic Control, IEEE Transactions on, vol. 58, no. 5, pp. 1332-1337, 2013.

[14] W. P. M. H. Heemels, M. C. F. Donkers, and A. R. Teel, "Periodic event-triggered control for linear systems," IEEE Transactions on Automatic Control, vol. 58, no. 4, pp. 847-861, 2013.

[15] P. Tabuada, "Event-triggered real-time scheduling of stabilizing control tasks," Automatic Control, IEEE Transactions on, vol. 52, no. 9, pp. 1680-1685, 2007.

[16] A. Anta and P. Tabuada, "To sample or not to sample: Self-triggered control for nonlinear systems," Automatic Control, IEEE Transactions on, vol. 55, no. 9, pp. 2030-2042, 2010.

[17] M. Mazo Jr, A. Anta, and P. Tabuada, "On self-triggered control for linear systems: Guarantees and complexity," in Control Conference (ECC), 2009 European. IEEE, 2009, pp. 3767-3772.

[18] D. V. Dimarogonas, E. Frazzoli, and K. H. Johansson, "Distributed event-triggered control for multi-agent systems," IEEE Transactions on Automatic Control, vol. 57, no. 5, pp. 1291-1297, 2012.

[19] G. S. Seyboth, D. V. Dimarogonas, and K. H. Johansson, "Event-based broadcasting for multi-agent average consensus," Automatica, vol. 49, no. 1 , pp. 245-252, 2013.
[20] J. Guerrero-Castellanos, J. Téllez-Guzmán, S. Durand, N. Marchand, J. Alvarez-Muñoz, and V. R. Gonzalez-Diaz, "Attitude stabilization of a quadrotor by means of event-triggered nonlinear control," Journal of Intelligent \& Robotic Systems, vol. 73, no. 1-4, pp. 123-135, 2014.

[21] J. F. Guerrero-Castellanos, N. Marchand, A. Hably, S. Lesecq, and J. Delamare, "Bounded attitude control of rigid bodies: Real-time experimentation to a quadrotor mini-helicopter," Control Engineering Practice, vol. 19, no. 8, pp. 790-797, 2011.

[22] M. D. Shuster, "A survey of attitude representations," Navigation, vol. 8, no. 9, pp. 439-517, 1993.

[23] R. Schlanbusch, A. Loria, and P. J. Nicklasson, "On the stability and stabilization of quaternion equilibria of rigid bodies," Automatica, vol. 48, no. 12, pp. 3135-3141, 2012.

[24] R. Sepulchre, M. Jankovic, and P. V. Kokotović, Constructive Nonlinear Control. London: Springler, 1997.

[25] A. Zavala, I. Fantoni, and R. Lozano, "Global stabilization of a pvtol aircraft model with bounded inputs," International Journal of Control, vol. 76 , no. 18, pp. 1833-1844, 2003. 